\title{
REFINEMENTS AND REVERSES OF FÉJER'S INEQUALITIES FOR CONVEX FUNCTIONS ON LINEAR SPACES
}

\begin{abstract}
In this paper, we establish some refinements and reverses of the celebrated Féjer's inequalities for the general case of functions defined on linear spaces. The obtained bounds are in terms of the Gâteaux lateral derivatives. Some applications for norms and semi-inner products in normed linear spaces are also provided.
\end{abstract}

Key words: Convex functions, Integral inequalities, Hermite-Hadamard inequality, Féjer's inequalities

2010 Mathematical Subject Classification: 26D15, 26D10

1. Introduction. Let $X$ be a real linear space, $x, y \in X, x \neq y$, and let $[x, y]:=\{(1-\lambda) x+\lambda y, \lambda \in[0,1]\}$ be the segment generated by $x$ and $y$. We consider the function $f:[x, y] \rightarrow \mathbb{R}$ and the attached function $\varphi_{(x, y)}:[0,1] \rightarrow \mathbb{R}, \varphi_{(x, y)}(t):=f[(1-t) x+t y], t \in[0,1]$.

It is well known that $f$ is convex on $[x, y]$ iff $\varphi(x, y)$ is convex on $[0,1]$, and the following lateral derivatives exist and satisfy

(i) $\varphi_{ \pm(x, y)}^{\prime}(s)=\nabla_{ \pm} f_{(1-s) x+s y}(y-x), s \in[0,1)$,

(ii) $\varphi_{+(x, y)}^{\prime}(0)=\nabla_{+} f_{x}(y-x)$,

(iii) $\varphi_{-(x, y)}^{\prime}(1)=\nabla_{-} f_{y}(y-x)$,

where $\nabla_{ \pm} f_{x}(y)$ are the Gâteaux lateral derivatives. Recall that

$$
\begin{aligned}
& \nabla_{+} f_{x}(y):=\lim _{h \rightarrow 0+} \frac{f(x+h y)-f(x)}{h}, x, y \in X, \\
& \nabla_{-} f_{x}(y):=\lim _{k \rightarrow 0-} \frac{f(x+k y)-f(x)}{k}, x, y \in X .
\end{aligned}
$$

(c) Petrozavodsk State University, 2020 
The following inequality is the well-known Hermite-Hadamard integral inequality for convex functions defined on a segment $[x, y] \subset X$ :

$$
f\left(\frac{x+y}{2}\right) \leqslant \int_{0}^{1} f[(1-t) x+t y] d t \leqslant \frac{f(x)+f(y)}{2},
$$

which easily follows by the classical Hermite-Hadamard inequality for the convex function $\varphi(x, y):[0,1] \rightarrow \mathbb{R}$

$$
\varphi_{(x, y)}\left(\frac{1}{2}\right) \leqslant \int_{0}^{1} \varphi_{(x, y)}(t) d t \leqslant \frac{\varphi_{(x, y)}(0)+\varphi_{(x, y)}(1)}{2} .
$$

For other related results, see the monograph on line [6]. For some recent results in linear spaces see [1], [2] and [8]- [11].

We have the following result [4], related to the first Hermite-Hadamard inequality in $(\mathrm{HH})$ :

Theorem 1. Let $X$ be a linear space, $x, y \in X, x \neq y$ and $f:[x, y] \rightarrow \mathbb{R}$ be a convex function on the segment $[x, y] \subset X$. Then, for any $s \in(0,1)$, one has the inequality

$$
\begin{gathered}
\frac{1}{2}\left[(1-s)^{2} \nabla_{+} f_{(1-s) x+s y}(y-x)-s^{2} \nabla_{-} f_{(1-s) x+s y}(y-x)\right] \leqslant \\
\leqslant \int_{0}^{1} f[(1-t) x+t y] d t-f[(1-s) x+s y] \leqslant \\
\leqslant \frac{1}{2}\left[(1-s)^{2} \nabla_{-} f_{y}(y-x)-s^{2} \nabla_{+} f_{x}(y-x)\right] .
\end{gathered}
$$

The constant $\frac{1}{2}$ is sharp in both inequalities. The second inequality also holds for $s=0$ or $s=1$.

If $f:[x, y] \rightarrow \mathbb{R}$ is as in Theorem 1 and Gâteaux differentiable at $c:=(1-\lambda) x+\lambda y, \lambda \in(0,1)$ along the direction $y-x$, then we have the inequality:

$$
\left(\frac{1}{2}-\lambda\right) \nabla f_{c}(y-x) \leqslant \int_{0}^{1} f[(1-t) x+t y] d t-f(c) .
$$


If $f$ is as in Theorem 1 , then

$$
\begin{aligned}
& 0 \leqslant \frac{1}{8}\left[\nabla_{+} f_{\frac{x+y}{2}}\right.\left.(y-x)-\nabla_{-} f_{\frac{x+y}{2}}(y-x)\right] \leqslant \\
& \leqslant \int_{0}^{1} f[(1-t) x+t y] d t-f\left(\frac{x+y}{2}\right) \leqslant \\
& \leqslant \frac{1}{8}\left[\nabla_{-} f_{y}(y-x)-\nabla_{+} f_{x}(y-x)\right] .
\end{aligned}
$$

The constant $\frac{1}{8}$ is sharp in both inequalities.

Also, we have the following result [5] related to the second HermiteHadamard inequality in $(\mathrm{HH})$ :

Theorem 2. Let $X$ be a linear space, $x, y \in X, x \neq y$ and $f:[x, y] \rightarrow \mathbb{R}$ be a convex function on the segment $[x, y] \subset X$. Then for any $s \in(0,1)$ one has the inequality

$$
\begin{gathered}
\frac{1}{2}\left[(1-s)^{2} \nabla_{+} f_{(1-s) x+s y}(y-x)-s^{2} \nabla_{-} f_{(1-s) x+s y}(y-x)\right] \leqslant \\
\leqslant(1-s) f(x)+s f(y)-\int_{0}^{1} f[(1-t) x+t y] d t \leqslant \\
\leqslant \frac{1}{2}\left[(1-s)^{2} \nabla_{-} f_{y}(y-x)-s^{2} \nabla_{+} f_{x}(y-x)\right] .
\end{gathered}
$$

The constant $\frac{1}{2}$ is sharp in both inequalities. The second inequality also holds for $s=0$ or $s=1$.

If $f:[x, y] \rightarrow \mathbb{R}$ is as in Theorem 2 and Gâteaux differentiable in $c:=(1-\lambda) x+\lambda y, \lambda \in(0,1)$ along the direction $y-x$, then we have the inequality:

$$
\left(\frac{1}{2}-\lambda\right) \nabla f_{c}(y-x) \leqslant(1-\lambda) f(x)+\lambda f(y)-\int_{0}^{1} f[(1-t) x+t y] d t .
$$

If $f$ is as in Theorem 2, then

$$
0 \leqslant \frac{1}{8}\left[\nabla_{+} f_{\frac{x+y}{2}}(y-x)-\nabla_{-} f_{\frac{x+y}{2}}(y-x)\right] \leqslant
$$




$$
\begin{aligned}
\leqslant \frac{f(x)+f(y)}{2} & -\int_{0}^{1} f[(1-t) x+t y] d t \leqslant \\
& \leqslant \frac{1}{8}\left[\nabla_{-} f_{y}(y-x)-\nabla_{+} f_{x}(y-x)\right] .
\end{aligned}
$$

The constant $\frac{1}{8}$ is sharp in both inequalities.

By the convexity of $f$, we have, for all $t \in[0,1]$, the inequality

$$
f\left(\frac{x+y}{2}\right) \leqslant \frac{f[(1-t) x+t y]+f[(1-t) y+t x]}{2} \leqslant \frac{f(x)+f(y)}{2} .
$$

If we multiply this inequality by $p:[0,1] \rightarrow[0, \infty)$, a Lebesgue integrable function on $[0,1]$, and integrate on $[0,1]$ over $t \in[0,1]$, then we get

$$
\begin{aligned}
& f\left(\frac{x+y}{2}\right) \int_{0}^{1} p(t) d t \leqslant \\
& \leqslant \frac{\int_{0}^{1} f[(1-t) x+t y] p(t) d t+\int_{0}^{1} f[(1-t) y+t x] p(t) d t}{2} \leqslant \frac{f(x)+f(y)}{2} \int_{0}^{1} p(t) d t .
\end{aligned}
$$

By changing the variable $s=1-t$, we get

$$
\int_{0}^{1} f[(1-t) y+t x] p(t) d t=\int_{0}^{1} f[s y+(1-s) x] p(1-s) d t
$$

and by (7) we obtain

$$
\begin{aligned}
f\left(\frac{x+y}{2}\right) \int_{0}^{1} p(t) d t \leqslant \int_{0}^{1} f[(1-t) x+t y] \breve{p}(t) d t \leqslant & \\
& \leqslant \frac{f(x)+f(y)}{2} \int_{0}^{1} p(t) d t
\end{aligned}
$$


where $\breve{p}(t):=\frac{1}{2}[p(t)+p(1-t)], t \in[0,1]$.

If $p$ is symmetric on $t[0,1]$, namely $p(t)=p(1-t)$ for $t \in[0,1]$, then (8) becomes the Féjer's inequality

$$
\begin{aligned}
f\left(\frac{x+y}{2}\right) \int_{0}^{1} p(t) d t \leqslant \int_{0}^{1} f[(1-t) x+t y] & p(t) d t \leqslant \\
& \leqslant \frac{f(x)+f(y)}{2} \int_{0}^{1} p(t) d t .
\end{aligned}
$$

Motivated by the above results, we establish in this paper some refinements and reverses of Féjer's inequalities (9). Some applications for norms and semi-inner products are also provided.

2. Refinements and Reverse Féjer Inequalities. We have:

Theorem 3. Let $f$ be an convex function on a convex set $C$ and $x, y \in C$ with $x \neq y$. If $p:[0,1] \rightarrow[0, \infty)$ is Lebesgue integrable and symmetric, namely $p(1-t)=p(t)$ for all $t \in[0,1]$, then

$$
\begin{aligned}
0 & \leqslant \frac{1}{2}\left[\nabla_{+} f_{\frac{x+y}{2}}(y-x)-\nabla_{-} f_{\frac{x+y}{2}}(y-x)\right] \int_{0}^{1}\left|t-\frac{1}{2}\right| p(t) d t \leqslant \\
& \leqslant \int_{0}^{1} f((1-t) x+t y) p(t) d t-f\left(\frac{x+y}{2}\right) \int_{0}^{1} p(t) d t \leqslant \\
& \leqslant \frac{1}{2}\left[\nabla_{-} f_{y}(y-x)-\nabla_{+} f_{x}(y-x)\right]\left(\int_{0}^{1}\left|t-\frac{1}{2}\right| p(t) d t\right) .
\end{aligned}
$$

Proof. Let $x, y \in C$, with $x \neq y$. Since $\varphi_{(x, y)}$ is differentiable everywhere on $[0,1]$ except a countable number of points, by using the integration by parts formula for the Lebesgue integral, we have

$$
\begin{aligned}
& \int_{1 / 2}^{1}\left(\int_{t}^{1} p(s) d s\right) \varphi_{(x, y)}^{\prime}(t) d t= \\
& \left.\quad=\left(\int_{t}^{1} p(s) d s\right) \varphi_{(x, y)}(t)\right]_{1 / 2}^{1}+\int_{1 / 2}^{1} p(t) \varphi_{(x, y)}(t) d t=
\end{aligned}
$$




$$
\begin{gathered}
=-\left(\int_{1 / 2}^{1} p(s) d s\right) \varphi_{(x, y)}(1 / 2)+\int_{1 / 2}^{1} p(t) \varphi_{(x, y)}(t) d t= \\
=-\left(\int_{1 / 2}^{1} p(s) d s\right) f\left(\frac{x+y}{2}\right)+\int_{1 / 2}^{1} p(t) \varphi_{(x, y)}(t) d t
\end{gathered}
$$

and

$$
\begin{aligned}
& \int_{0}^{1 / 2}\left(\int_{0}^{t} p(s) d s\right) \varphi_{(x, y)}^{\prime}(t) d t= \\
&=\left.\left(\int_{0}^{t} p(s) d s\right) \varphi_{(x, y)}(t)\right]_{0}^{1 / 2}-\int_{0}^{1 / 2} p(t) \varphi_{(x, y)}(t) d t= \\
&=\left(\int_{0}^{1 / 2} p(s) d s\right) \varphi_{(x, y)}(1 / 2)-\int_{0}^{1 / 2} p(t) \varphi(x, y)(t) d t= \\
&=\left(\int_{0}^{1 / 2} p(s) d s\right) f\left(\frac{x+y}{2}\right)-\int_{0} p(t) \varphi_{(x, y)}(t) d t .
\end{aligned}
$$

Subtracting the second identity from the first, we get

$$
\begin{aligned}
& \int_{1 / 2}^{1}\left(\int_{t}^{1} p(s) d s\right) \varphi_{(x, y)}^{\prime}(t) d t-\int_{0}^{1 / 2}\left(\int_{0}^{t} p(s) d s\right) \varphi_{t(x, y)}^{\prime}(t) d t= \\
&=\int_{1 / 2}^{1} p(t) \varphi(x, y)(t) d t+\int_{0}^{1 / 2} p(t) \varphi(x, y)(t) d t- \\
&-\left(\int_{1 / 2}^{1} p(s) d s\right) f\left(\frac{x+y}{2}\right)-\left(\int_{0}^{1 / 2} p(s) d s\right) f\left(\frac{x+y}{2}\right) .
\end{aligned}
$$

By the symmetry of $p$ we get

$$
\int_{1 / 2}^{1} p(s) d s=\int_{0}^{1 / 2} p(s) d s=\frac{1}{2} \int_{0}^{1} p(s) d s
$$


and, then, we get the following identity, which is of interest itself:

$$
\begin{aligned}
& \int_{0}^{1} p(t) \varphi_{(x, y)}(t) d t-\int_{0}^{1} p(s) d s f\left(\frac{x+y}{2}\right)= \\
& =\int_{1 / 2}^{1}\left(\int_{t}^{1} p(s) d s\right) \varphi_{(x, y)}^{\prime}(t) d t-\int_{0}^{1 / 2}\left(\int_{0}^{t} p(s) d s\right) \varphi_{(x, y)}^{\prime}(t) d t .
\end{aligned}
$$

By the convexity of $\varphi_{(x, y)}$ on $[0,1]$, we have

$$
\nabla_{+} f_{x}(y-x)=\varphi_{+(x, y)}^{\prime}(0) \leqslant \varphi_{(x, y)}^{\prime}(t) \leqslant \varphi_{-(x, y)}^{\prime}\left(\frac{1}{2}\right)=\nabla_{-} f_{\frac{x+y}{2}}(y-x),
$$

for almost every $t \in[0,1 / 2]$; also

$$
\nabla_{+} f_{\frac{x+y}{2}}(y-x)=\varphi_{+(x, y)}^{\prime}\left(\frac{1}{2}\right) \leqslant \varphi_{(x, y)}^{\prime}(t) \leqslant \varphi_{-(x, y)}^{\prime}(1)=\nabla_{-} f_{y}(y-x)
$$

for almost every $t \in[1 / 2,1]$.

This implies

$$
\begin{aligned}
\left(\int_{0}^{t} p(s) d s\right) \nabla_{+} f_{x}(y-x) \leqslant\left(\int_{0}^{t} p(s) d s\right) \varphi_{(x, y)}^{\prime}(t) \leqslant & \\
& \leqslant\left(\int_{0}^{t} p(s) d s\right) \nabla_{-} f_{\frac{x+y}{2}}(y-x),
\end{aligned}
$$

for almost every $t \in[0,1 / 2]$ and

$$
\begin{aligned}
\left(\int_{t}^{1} p(s) d s\right) \nabla_{+} f_{\frac{x+y}{2}}(y-x) \leqslant\left(\int_{t}^{1} p(s) d s\right) \varphi_{(x, y)}^{\prime}(t) \leqslant & \\
& \leqslant\left(\int_{t}^{1} p(s) d s\right) \nabla_{-} f_{y}(y-x),
\end{aligned}
$$

for almost every $t \in[1 / 2,1]$. 
By integrating these two inequalities on the corresponding intervals, we obtain

$$
\begin{aligned}
\int_{1 / 2}^{1}\left(\int_{t}^{1} p(s) d s\right) d t \nabla_{+} f_{\frac{x+y}{2}}(y-x) & \leqslant \int_{1 / 2}^{1}\left(\int_{t}^{1} p(s) d s\right) \varphi_{(x, y)}^{\prime}(t) d t \leqslant \\
& \leqslant \int_{1 / 2}^{1}\left(\int_{t}^{1} p(s) d s\right) d t \nabla_{-} f_{y}(y-x)
\end{aligned}
$$

and

$$
\begin{aligned}
-\int_{0}^{1 / 2}\left(\int_{0}^{t} p(s) d s\right) d t \nabla_{-} f_{\frac{x+y}{2}}(y-x) & \leqslant-\int_{0}^{1 / 2}\left(\int_{0}^{t} p(s) d s\right) \varphi_{(x, y)}^{\prime}(t) d t \leqslant \\
& \leqslant-\int_{0}^{1 / 2}\left(\int_{0}^{t} p(s) d s\right) d t \nabla_{+} f_{x}(y-x) .
\end{aligned}
$$

Adding these inequalities, we get

$$
\begin{gathered}
\int_{1 / 2}^{1}\left(\int_{t}^{1} p(s) d s\right) d t \nabla_{+} f_{\frac{x+y}{2}}(y-x)-\int_{0}^{1 / 2}\left(\int_{0}^{t} p(s) d s\right) d t \nabla_{-} f_{\frac{x+y}{2}}(y-x) \leqslant \\
\leqslant \int_{1 / 2}^{1}\left(\int_{t}^{1} p(s) d s\right) \varphi_{(x, y)}^{\prime}(t) d t-\int_{0}^{1 / 2}\left(\int_{0}^{t} p(s) d s\right) \varphi_{(x, y)}^{\prime}(t) d t \leqslant \\
\leqslant \int_{1 / 2}^{1}\left(\int_{t}^{1} p(s) d s\right) d t \nabla_{-} f_{y}(y-x)-\int_{0}^{1 / 2}\left(\int_{0}^{t} p(s) d s\right) d t \nabla_{+} f_{x}(y-x)
\end{gathered}
$$

for all $x, y \in C$, with $x \neq y$.

Further, integrating by parts in the Lebesgue integral, we have

$$
\left.\int_{1 / 2}^{1}\left(\int_{t}^{1} p(s) d s\right) d t=\left(\int_{t}^{1} p(s) d s\right) t\right]_{1 / 2}^{1}+\int_{1 / 2}^{1} t p(t) d t=
$$




$$
=\int_{1 / 2}^{1} t p(t) d t-\frac{1}{2} \int_{1 / 2}^{1} p(s) d s=\int_{1 / 2}^{1}\left(t-\frac{1}{2}\right) p(t) d t
$$

and

$$
\begin{aligned}
\int_{0}^{1 / 2}\left(\int_{0}^{t} p(s) d s\right) d t= & \left.\left(\int_{0}^{t} p(s) d s\right) t\right]_{0}^{1 / 2}-\int_{0}^{1 / 2} p(t) t d t \\
= & \frac{1}{2} \int_{0}^{1 / 2} p(s) d s-\int_{0}^{1 / 2} p(t) t d t=\int_{0}^{1 / 2}\left(\frac{1}{2}-t\right) p(t) d t .
\end{aligned}
$$

We have

$$
\int_{0}^{1}\left|t-\frac{1}{2}\right| p(t) d t=\int_{1 / 2}^{1}\left(t-\frac{1}{2}\right) p(t) d t+\int_{0}^{1 / 2}\left(\frac{1}{2}-t\right) p(t) d t .
$$

Since $p$ is symmetric on $[0,1]$, hence, by changing the variable $s=1-t$, we have

$$
\begin{aligned}
\int_{0}^{1 / 2}\left(\frac{1}{2}-t\right) p(t) d t & =\int_{1 / 2}^{1}\left(s-\frac{1}{2}\right) p(1-s) d s= \\
& =\int_{1 / 2}^{1}\left(s-\frac{1}{2}\right) p(s) d s=\int_{1 / 2}^{1}\left(t-\frac{1}{2}\right) p(t) d t
\end{aligned}
$$

this shows that

$$
\int_{1 / 2}^{1}\left(t-\frac{1}{2}\right) p(t) d t=\int_{0}^{1 / 2}\left(\frac{1}{2}-t\right) p(t) d t=\frac{1}{2} \int_{0}^{1}\left|t-\frac{1}{2}\right| p(t) d t .
$$

By utilising (12), (13), (14), and (15) we obtain (10).

Remark. Putting $p \equiv 1$ in (10), we recapture the result (3). Taking $p(t)=\left|t-\frac{1}{2}\right|, t \in[0,1]$, we get 


$$
\begin{aligned}
& 0 \leqslant \frac{1}{24}\left[\nabla_{+} f_{\frac{x+y}{2}}(y-x)-\nabla_{-} f_{\frac{x+y}{2}}(y-x)\right] \leqslant \\
& \leqslant \int_{0}^{1} f((1-t) x+t y)\left|t-\frac{1}{2}\right| d t-\frac{1}{4} f\left(\frac{x+y}{2}\right) \leqslant \\
& \leqslant \frac{1}{24}\left[\nabla_{-} f_{y}(y-x)-\nabla_{+} f_{x}(y-x)\right] .
\end{aligned}
$$

We also have:

Theorem 4. Let $f$ be an convex function on a convex set $C$ and $x, y \in C$, with $x \neq y$. If $p:[0,1] \rightarrow[0, \infty)$ is Lebesgue integrable and symmetric, namely $p(1-t)=p(t)$ for all $t \in[0,1]$, then

$$
\begin{aligned}
0 & \leqslant \frac{1}{2}\left[\nabla_{+} f_{\frac{x+y}{2}}(y-x)-\nabla_{-} f_{\frac{x+y}{2}}(y-x)\right] \int_{0}^{1}\left(\frac{1}{2}-\left|t-\frac{1}{2}\right|\right) p(t) d t \leqslant \\
& \leqslant \frac{f(x)+f(y)}{2} \int_{0}^{1} p(t) d t-\int_{0}^{1} f((1-t) x+t y) p(t) d t \leqslant \\
& \leqslant \frac{1}{2}\left[\nabla_{-} f_{y}(y-x)-\nabla_{+} f_{x}(y-x)\right] \int_{0}^{1}\left(\frac{1}{2}-\left|t-\frac{1}{2}\right|\right) p(t) d t
\end{aligned}
$$

Proof. Using the integration by parts for the Lebesgue integral, we have

$$
\begin{aligned}
& \int_{0}^{1}\left(\int_{0}^{t} p(s) d s-\frac{1}{2} \int_{0}^{1} p(s) d s\right) \varphi_{(x, y)}^{\prime}(t) d t= \\
& \left.\quad=\left(\int_{0}^{t} p(s) d s-\frac{1}{2} \int_{0}^{1} p(s) d s\right) \varphi_{(x, y)}(t)\right]_{0}^{1}-\int_{0}^{1} p(t) \varphi_{(x, y)}(t) d t= \\
& =\left(\int_{0}^{1} p(s) d s-\frac{1}{2} \int_{0}^{1} p(s) d s\right) \varphi_{(x, y)}(1)+\left(\frac{1}{2} \int_{0}^{1} p(s) d s\right) \varphi_{(x, y)}(0)- \\
& -\int_{0}^{1} p(t) \varphi_{(x, y)}(t) d t=\left(\int_{0}^{1} p(t) d t\right) \frac{f(x)+f(y)}{2}-\int_{0}^{1} p(t) \varphi_{(x, y)}(t) d t .
\end{aligned}
$$


We also have

$$
\begin{aligned}
& \left.\int_{0}^{1} \int_{0}^{t} p(s) d s-\frac{1}{2} \int_{0}^{1} p(s) d s\right) \varphi_{(x, y)}^{\prime}(t) d t= \\
& =\int_{0}^{1}\left(\int_{0}^{t} p(s) d s-\int_{0}^{1 / 2} p(s) d s\right) \varphi_{(x, y)}^{\prime}(t) d t= \\
& =\int_{0}^{1 / 2}\left(\int_{0}^{t} p(s) d s-\int_{0}^{1 / 2} p(s) d s\right) \varphi_{(x, y)}^{\prime}(t) d t+ \\
& +\int_{1 / 2}^{1}\left(\int_{0}^{t} p(s) d s-\int_{0}^{1 / 2} p(s) d s\right) \varphi_{(x, y)}^{\prime}(t) d t= \\
& =\int_{1 / 2}^{1}\left(\int_{0}^{t} p(s) d s-\int_{0}^{1 / 2} p(s) d s\right) \varphi_{(x, y)-}^{\prime}(t) d t- \\
& -\int_{0}^{1 / 2}\left(\int_{0}^{1 / 2} p(s) d s-\int_{0}^{t} p(s) d s\right) \varphi_{(x, y)}^{\prime}(t) d t .
\end{aligned}
$$

Observe that

$$
\begin{aligned}
& \int_{0}^{t} p(s) d s-\int_{0}^{1 / 2} p(s) d s \geqslant 0 \text { for } t \in[1 / 2,1], \\
& \int_{0}^{1 / 2} p(s) d s-\int_{0}^{t} p(s) d s \geqslant 0 \text { for } t \in[0,1 / 2] .
\end{aligned}
$$

By the convexity of $\varphi_{(x, y)}$ on the interval $[0,1]$, we deduce

$$
\begin{aligned}
& \int_{1 / 2}^{1}\left(\int_{0}^{t} p(s) d s-\int_{0}^{1 / 2} p(s) d s\right) d t \nabla_{+} f_{\frac{x+y}{2}}(y-x) \leqslant \\
& \leqslant \int_{1 / 2}^{1}\left(\int_{0}^{t} p(s) d s-\int_{0}^{1 / 2} p(s) d s\right) \varphi_{(x, y)}^{\prime}(t) d t \leqslant
\end{aligned}
$$




$$
\leqslant \int_{1 / 2}^{1}\left(\int_{0}^{t} p(s) d s-\int_{0}^{1 / 2} p(s) d s\right) d t \nabla_{-} f_{y}(y-x)
$$

and

$$
\begin{aligned}
& -\int_{0}^{1 / 2}\left(\int_{0}^{1 / 2} p(s) d s-\int_{0}^{t} p(s) d s\right) d t \nabla_{-} f_{\frac{x+y}{2}}(y-x) \leqslant \\
& \leqslant-\int_{0}^{1 / 2}\left(\int_{0}^{1 / 2} p(s) d s-\int_{0}^{t} p(s) d s\right) \varphi_{(x, y)}^{\prime}(t) d t \leqslant \\
& \leqslant-\int_{0}^{1 / 2}\left(\int_{0}^{1 / 2} p(s) d s-\int_{0}^{t} p(s) d s\right) d t \nabla_{+} f_{x}(y-x) .
\end{aligned}
$$

Adding these inequalities, we get

$$
\begin{aligned}
\int_{1 / 2}^{1}\left(\int_{0}^{t} p(s) d s-\int_{0}^{1 / 2} p(s) d s\right) d t \nabla_{+} f_{\frac{x+y}{2}}(y-x)- \\
-\int_{0}^{1 / 2}\left(\int_{0}^{1 / 2} p(s) d s-\int_{0}^{t} p(s) d s\right) d t \nabla_{-} f_{\frac{x+y}{2}}(y-x) \leqslant \\
\leqslant \int_{1 / 2}^{1}\left(\int_{0}^{t} p(s) d s-\int_{0}^{1 / 2} p(s) d s\right) \varphi_{(x, y)}^{\prime}(t) d t- \\
-\int_{1 / 2}^{1}\left(\int_{0}^{t} p(s) d s-\int_{0}^{1 / 2} p(s) d s\right) \varphi_{(x, y)}^{\prime}(t) d t \leqslant \\
\leqslant \int_{1 / 2}^{1}\left(\int_{0}^{1 / 2} p(s) d s-\int_{0}^{t / 2} p(s) d s\right) d t \nabla_{-} f_{y}(y-x)- \\
-\int_{0}^{t}\left(\int_{0}^{t} p(s) d s-\int_{0}^{1 / 2} p(s) d s\right) d t \nabla_{+} f_{x}(y-x) .
\end{aligned}
$$


Since

$$
\begin{aligned}
& \int_{1 / 2}^{1}\left(\int_{0}^{t} p(s) d s-\int_{0}^{1 / 2} p(s) d s\right) d t= \\
= & \int_{1 / 2}^{1}\left(\int_{0}^{t} p(s) d s\right) d t-\frac{1}{2} \int_{0}^{1 / 2} p(s) d s= \\
= & \left.\left.\int_{0}^{t} p(s) d s\right) t\right]_{1 / 2}^{1}-\int_{1 / 2}^{1} t p(t) d t-\frac{1}{2} \int_{0}^{1 / 2} p(s) d s= \\
= & \int_{0}^{1} p(s) d s-\frac{1}{2} \int_{0}^{1 / 2} p(s) d s-\int_{1 / 2}^{1} t p(t) d t-\frac{1}{2} \int_{0}^{1 / 2} p(s) d s= \\
= & \int_{0}^{1} p(s) d s-\int_{0}^{1 / 2} p(s) d s-\int_{1 / 2}^{1} t p(t) d t= \\
= & \int_{1 / 2}^{1} p(s) d s-\int_{1 / 2}^{1} t p(t) d t=\int_{1 / 2}^{1}(1-t) p(t) d t
\end{aligned}
$$

and

$$
\begin{aligned}
& \int_{0}^{1 / 2}\left(\int_{0}^{1 / 2} p(s) d s-\int_{0}^{t} p(s) d s\right) d t= \\
& =\frac{1}{2} \int_{0}^{1 / 2} p(s) d s-\int_{0}^{1 / 2}\left(\int_{0}^{t} p(s) d s\right) d t= \\
& \left.=\frac{1}{2} \int_{0}^{1 / 2} p(s) d s-\left(\left(\int_{0}^{t} p(s) d s\right) t\right]_{0}^{1 / 2}-\int_{0}^{1 / 2} t p(t) d t\right)= \\
& =\frac{1}{2} \int_{0}^{1 / 2} p(s) d s-\frac{1}{2} \int_{0}^{1 / 2} p(s) d s+\int_{0}^{1 / 2} t p(t) d t=\int_{0}^{1 / 2} t p(t) d t .
\end{aligned}
$$


If we change the variable $s=1-t$, then

$$
\begin{array}{r}
\int_{0}^{1 / 2} t p(t) d t=-\int_{1}^{1 / 2}(1-s) p(1-s) d s=\int_{1 / 2}^{1}(1-s) p(1-s) d s= \\
=\int_{1 / 2}^{1}(1-s) p(s) d s .
\end{array}
$$

Therefore

$$
\begin{aligned}
& \frac{1}{2} \int_{0}^{1}\left(\frac{1}{2}-\left|t-\frac{1}{2}\right|\right) p(t) d t= \\
& =\frac{1}{2} \int_{0}^{1 / 2}\left(\frac{1}{2}-\left|t-\frac{1}{2}\right|\right) p(t) d t+\frac{1}{2} \int_{1 / 2}^{1}\left(\frac{1}{2}-\left|t-\frac{1}{2}\right|\right) p(t) d t= \\
& =\frac{1}{2} \int_{0}^{1 / 2}\left(\frac{1}{2}-\frac{1}{2}+t\right) p(t) d t+\frac{1}{2} \int_{1 / 2}^{1}\left(\frac{1}{2}-t+\frac{1}{2}\right) p(t) d t= \\
& =\frac{1}{2} \int_{0}^{1 / 2} t p(t) d t+\frac{1}{2} \int_{1 / 2}^{1}(1-t) p(t) d t=\int_{0}^{1 / 2} t p(t) d t \text { (by 20) }
\end{aligned}
$$

and by (18), (13), (14) and (19) we get the desired result (17).

Remark. If we put $p \equiv 1$ in (17), then we recapture the earlier result (6). If we take $p(t)=\left|t-\frac{1}{2}\right|, t \in[0,1]$ in (17), then we get

$$
\begin{aligned}
0 & \leqslant \frac{1}{48}\left[\nabla_{+} f_{\frac{x+y}{2}}(y-x)-\nabla_{-} f_{\frac{x+y}{2}}(y-x)\right] \leqslant \\
& \leqslant \frac{f(x)+f(y)}{8}-\int_{0}^{1} f((1-t) x+t y)\left|t-\frac{1}{2}\right| d t \leqslant \\
& \leqslant \frac{1}{48}\left[\nabla_{-} f_{y}(y-x)-\nabla_{+} f_{x}(y-x)\right] .
\end{aligned}
$$

3. Examples for Norms. Now, assume that $(X,\|\cdot\|)$ is a normed linear space. The function $f_{0}(s)=\frac{1}{2}\|x\|^{2}, x \in X$ is convex and thus the following limits exist: 


$$
\begin{aligned}
\text { (iv) }\langle x, y\rangle_{s}:=\nabla_{+} f_{0, y}(x)=\lim _{t \rightarrow 0+} \frac{\|y+t x\|^{2}-\|y\|^{2}}{2 t} ; \\
\text { (v) }\langle x, y\rangle_{i}:=\nabla_{-} f_{0, y}(x)=\lim _{s \rightarrow 0-} \frac{\|y+s x\|^{2}-\|y\|^{2}}{2 s} ;
\end{aligned}
$$

for any $x, y \in X$. They are called the lower and the upper semi-inner products associated to the norm $\|\cdot\|$.

For the sake of completeness, we list here some of the main properties of these mappings that will be used in the sequel (see, for example, [2] or [7]); assume that $p, q \in\{s, i\}$ and $p \neq q$ :

(a) $\langle x, x\rangle_{p}=\|x\|^{2}$ for all $x \in X$;

(aa) $\langle\alpha x, \beta y\rangle_{p}=\alpha \beta\langle x, y\rangle_{p}$ if $\alpha, \beta \geqslant 0$ and $x, y \in X$;

(aaa) $\left|\langle x, y\rangle_{p}\right| \leqslant\|x\|\|y\|$ for all $x, y \in X$;

(av) $\langle\alpha x+y, x\rangle_{p}=\alpha\langle x, x\rangle_{p}+\langle y, x\rangle_{p}$ if $x, y \in X$ and $\alpha \in \mathbb{R}$;

(v) $\langle-x, y\rangle_{p}=-\langle x, y\rangle_{q}$ for all $x, y \in X$;

(va) $\langle x+y, z\rangle_{p} \leqslant\|x\|\|z\|+\langle y, z\rangle_{p}$ for all $x, y, z \in X$;

(vaa) The mapping $\langle\cdot, \cdot\rangle_{p}$ is continuous and subadditive (superadditive) in the first variable for $p=s \quad($ or $p=i)$;

(vaaa) The normed linear space $(X,\|\cdot\|)$ is smooth at the point $x_{0} \in X \backslash\{0\}$ if and only if $\left\langle y, x_{0}\right\rangle_{s}=\left\langle y, x_{0}\right\rangle_{i}$ for all $y \in X$; in general, $\langle y, x\rangle_{i} \leqslant\langle y, x\rangle_{s}$ for all $x, y \in X$

(ax) If the norm $\|\cdot\|$ is induced by an inner product $\langle\cdot, \cdot\rangle$, then $\langle y, x\rangle_{i}=\langle y, x\rangle=\langle y, x\rangle_{s}$ for all $x, y \in X$.

The function $f_{r}(x)=\|x\|^{r}(x \in X$ and $1 \leqslant r<\infty)$ is also convex. Therefore, the following limits, which are related to the superior (inferior) semi-inner products,

$$
\begin{aligned}
\nabla_{ \pm} f_{r, y}(x) & :=\lim _{t \rightarrow 0 \pm} \frac{\|y+t x\|^{r}-\|y\|^{r}}{t}= \\
& =r\|y\|^{r-1} \lim _{t \rightarrow 0 \pm} \frac{\|y+t x\|-\|y\|}{t}=r\|y\|^{r-2}\langle x, y\rangle_{s(i)}
\end{aligned}
$$

exist for all $x, y \in X$ whenever $r \geqslant 2$; otherwise, they exist for any $x \in X$ and nonzero $y \in X$. In particular, if $r=1$, then the following limits

$$
\nabla_{ \pm} f_{1, y}(x):=\lim _{t \rightarrow 0 \pm} \frac{\|y+t x\|-\|y\|}{t}=\frac{\langle x, y\rangle_{s(i)}}{\|y\|}
$$


exist for $x, y \in X$ and $y \neq 0$.

If we write the inequalities (10) for the function $f_{r}(x)=\|x\|^{r}(x \in X$ and $1 \leqslant r<\infty)$, we get

$$
\begin{aligned}
0 & \leqslant \frac{1}{2} r\left\|\frac{x+y}{2}\right\|^{r-2}\left[\left\langle y-x, \frac{x+y}{2}\right\rangle_{s}-\left\langle y-x, \frac{x+y}{2}\right\rangle_{i}\right] \times \\
& \times \int_{0}^{1}\left|t-\frac{1}{2}\right| p(t) d t \leqslant \\
& \leqslant \int_{0}^{1}\|(1-t) x+t y\|^{r} p(t) d t-\left\|\frac{x+y}{2}\right\|^{r} \int_{0}^{1} p(t) d t \leqslant \\
& \leqslant \frac{1}{2} r\left[\|y\|^{r-2}\langle y-x, y\rangle_{s}-\|x\|^{r-2}\langle y-x, x\rangle_{i}\right] \times \\
& \times \int_{0}^{1}\left|t-\frac{1}{2}\right| p(t) d t,
\end{aligned}
$$

for any Lebesgue-integrable and symmetric function $p:[0,1] \rightarrow[0, \infty)$.

If $r \geqslant 2$, the inequality (22) holds for any $x, y \in X$. If $r \in[1,2)$, the inequality (22) holds for any $x, y \in X$ with $x, y, x+y \neq 0$.

If we take $r=2$, we get the simpler inequality

$$
\begin{aligned}
0 & \leqslant\left[\left\langle y-x, \frac{x+y}{2}\right\rangle_{s}-\left\langle y-x, \frac{x+y}{2}\right\rangle_{i}\right] \int_{0}^{1}\left|t-\frac{1}{2}\right| p(t) d t \leqslant \\
& \leqslant \int_{0}^{1}\|(1-t) x+t y\|^{2} p(t) d t-\left\|\frac{x+y}{2}\right\|^{2} \int_{0}^{1} p(t) d t \leqslant \\
& \leqslant\left[\langle y-x, y\rangle_{s}-\langle y-x, x\rangle_{i}\right] \int_{0}^{1}\left|t-\frac{1}{2}\right| p(t) d t,
\end{aligned}
$$

for any $x, y \in X$.

If we write the inequalities (17) for the function $f_{r}(x)=\|x\|^{r}(x \in X$ and $1 \leqslant r<\infty)$, for any Lebesgue integrable and symmetric function $p:[0,1] \rightarrow[0, \infty)$, we get

$$
0 \leqslant \frac{1}{2} r\left\|\frac{x+y}{2}\right\|^{r-2}\left[\left\langle y-x, \frac{x+y}{2}\right\rangle_{s}-\left\langle y-x, \frac{x+y}{2}\right\rangle_{i}\right] \times
$$




$$
\begin{aligned}
& \times \int_{0}^{1}\left(\frac{1}{2}-\left|t-\frac{1}{2}\right|\right) p(t) d t \leqslant \\
& \leqslant \frac{\|x\|^{r}+\|y\|^{r}}{2} \int_{0}^{1} p(t) d t-\int_{0}^{1}\|(1-t) x+t y\|^{r} p(t) d t \leqslant \\
& \leqslant \frac{1}{2} r\left[\|y\|^{r-2}\langle y-x, y\rangle_{s}-\|x\|^{r-2}\langle y-x, x\rangle_{i}\right] \times \\
& \times \int_{0}^{1}\left(\frac{1}{2}-\left|t-\frac{1}{2}\right|\right) p(t) d t .
\end{aligned}
$$

If $r \geqslant 2$, the inequality (24) holds for any $x, y \in X$. If $r \in[1,2)$, the inequality (24) holds for any $x, y \in X$ with $x, y, x+y \neq 0$.

If $(H ;\langle\cdot, \cdot\rangle)$ is a real inner product space and $p:[0,1] \rightarrow[0, \infty)$ a Lebesgue integrable and symmetric function on $[0,1]$, then from $(22)$ we have

$$
\begin{aligned}
0 & \leqslant \int_{0}^{1}\|(1-t) x+t y\|^{r} p(t) d t-\left\|\frac{x+y}{2}\right\|^{r} \int_{0}^{1} p(t) d t \leqslant \\
& \leqslant \frac{1}{2} r\left\langle y-x,\|y\|^{r-2} y-\|x\|^{r-2} x\right\rangle \int_{0}^{1}\left|t-\frac{1}{2}\right| p(t) d t
\end{aligned}
$$

while from (24) we get

$$
\begin{aligned}
0 & \leqslant \frac{\|x\|^{r}+\|y\|^{r}}{2} \int_{0}^{1} p(t) d t-\int_{0}^{1}\|(1-t) x+t y\|^{r} p(t) d t \leqslant \\
& \leqslant \frac{1}{2} r\left\langle y-x,\|y\|^{r-2} y-\|x\|^{r-2} x\right\rangle \int_{0}^{1}\left(\frac{1}{2}-\left|t-\frac{1}{2}\right|\right) p(t) d t .
\end{aligned}
$$

In particular, for $r=2$, we derive the simpler inequalities

$$
0 \leqslant \int_{0}^{1}\|(1-t) x+t y\|^{2} p(t) d t-\left\|\frac{x+y}{2}\right\|^{2} \int_{0}^{1} p(t) d t \leqslant
$$




$$
\leqslant\|y-x\|^{2} \int_{0}^{1}\left|t-\frac{1}{2}\right| p(t) d t
$$

while from (24) we get

$$
\begin{aligned}
0 & \leqslant \frac{\|x\|^{2}+\|y\|^{2}}{2} \int_{0}^{1} p(t) d t-\int_{0}^{1}\|(1-t) x+t y\|^{2} p(t) d t \leqslant \\
& \leqslant\|y-x\|^{2} \int_{0}^{1}\left(\frac{1}{2}-\left|t-\frac{1}{2}\right|\right) p(t) d t
\end{aligned}
$$

for all $x, y \in H$.

3. Examples for Functions of Several Variables. Now, let $\Omega \subset \mathbb{R}^{n}$ be an open convex set in $\mathbb{R}^{n}$. If $F: \Omega \rightarrow \mathbb{R}$ is a differentiable convex function on $\Omega$, then, obviously, for any $\bar{c} \in \Omega$ we have

$$
\nabla F_{\bar{c}}(\bar{y})=\sum_{i=1}^{n} \frac{\partial F(\bar{c})}{\partial x_{i}} \cdot y_{i}, \quad \bar{y}=\left(y_{1}, \ldots, y_{n}\right) \in \mathbb{R}^{n},
$$

where $\frac{\partial F}{\partial x_{i}}$ are the partial derivatives of $F$ with respect to the variable $x_{i}$ $(i=1, \ldots, n)$.

Using the inequalities (10), we get, for all $\bar{a}, \bar{b} \in \Omega$ and a Lebesgue integrable and symmetric function $p:[0,1] \rightarrow[0, \infty)$ on $[0,1]$, that

$$
\begin{aligned}
0 & \leqslant \int_{0}^{1} F((1-t) \bar{a}+t \bar{b}) p(t) d t-F\left(\frac{\bar{a}+\bar{b}}{2}\right) \int_{0}^{1} p(t) d t \leqslant \\
& \leqslant \frac{1}{2}\left(\int_{0}^{1}\left|t-\frac{1}{2}\right| p(t) d t\right) \sum_{i=1}^{n}\left(\frac{\partial F(\bar{b})}{\partial x_{i}}-\frac{\partial F(\bar{a})}{\partial x_{i}}\right)\left(b_{i}-a_{i}\right)
\end{aligned}
$$

and by (17) we obtain

$$
\begin{aligned}
0 & \leqslant \frac{F(\bar{a})+F(\bar{b})}{2} \int_{0}^{1} p(t) d t-\int_{0}^{1} F((1-t) \bar{a}+t \bar{b}) p(t) d t \leqslant \\
& \leqslant \frac{1}{2} \int_{0}^{1}\left(\frac{1}{2}-\left|t-\frac{1}{2}\right|\right) p(t) d t \sum_{i=1}^{n}\left(\frac{\partial F(\bar{b})}{\partial x_{i}}-\frac{\partial F(\bar{a})}{\partial x_{i}}\right)\left(b_{i}-a_{i}\right) .
\end{aligned}
$$


For $p \equiv 1$, we recapture the results obtained in [4] and [5], while for $p(t)=\left|t-\frac{1}{2}\right|, t \in[0,1]$ we get

$$
\begin{aligned}
0 & \leqslant \int_{0}^{1} F((1-t) \bar{a}+t \bar{b})\left|t-\frac{1}{2}\right| d t-\frac{1}{4} F\left(\frac{\bar{a}+\bar{b}}{2}\right) \leqslant \\
& \leqslant \frac{1}{24} \sum_{i=1}^{n}\left(\frac{\partial F(\bar{b})}{\partial x_{i}}-\frac{\partial F(\bar{a})}{\partial x_{i}}\right)\left(b_{i}-a_{i}\right)
\end{aligned}
$$

and

$$
\begin{aligned}
0 & \leqslant \frac{F(\bar{a})+F(\bar{b})}{8}-\int_{0}^{1} F((1-t) \bar{a}+t \bar{b})\left|t-\frac{1}{2}\right| d t \leqslant \\
& \leqslant \frac{1}{48} \sum_{i=1}^{n}\left(\frac{\partial F(\bar{b})}{\partial x_{i}}-\frac{\partial F(\bar{a})}{\partial x_{i}}\right)\left(b_{i}-a_{i}\right)
\end{aligned}
$$

for all $\bar{a}, \bar{b} \in \Omega$.

Acknowledgment. The author would like to thank the anonymous referee for valuable suggestions that helped to improve the manuscript.

\section{References}

[1] Alomari M., Darus M. Refinements of s-Orlicz convex functions in normed linear spaces. Int. J. Contemp. Math. Sci., 2008, vol. 3, no. 29-32, pp. $1569-1594$.

[2] Ciorănescu I. Geometry of Banach Spaces, Duality Mappings and Nonlinear Problems, Kluwer Academic Publishers, Dordrecht, 1990.

[3] Chen X. New convex functions in linear spaces and Jensen's discrete inequality. J. Inequal. Appl., 2013, vol. 2013:472, 8 pp.

DOI: https://doi.org/10.1186/1029-242x-2013-472

[4] Dragomir S. S. An inequality improving the first Hermite-Hadamard inequality for convex functions defined on linear spaces and applications for semi-inner products, J. Inequal. Pure \& Appl. Math., 2002, vol. 3, no. 2, Article 31.

https://www.emis.de/journals/JIPAM/article183.html?sid=183

[5] Dragomir S. S. An inequality improving the second Hermite-Hadamard inequality for convex functions defined on linear spaces and applications for semi-inner products, J. Inequal. Pure \& Appl. Math., 2002, vol. 3, no. 3, Article 35.

https://www.emis.de/journals/JIPAM/article187.html?sid=187 
[6] Dragomir S. S., Pearce C. E. M. Selected Topics on Hermite-Hadamard Inequalities and Applications, RGMIA Monographs, Victoria University, 2000. https://rgmia.org/monographs/hermite_hadamard.html

[7] Dragomir S. S. Semi-inner Products and Applications. Nova Science Publishers, Inc., Hauppauge, NY, 2004. x+222 pp. ISBN: 1-59033-947-9.

[8] Khan M. A., Khalid S., Pečarić J. Improvement of Jensen's inequality in terms of Gâteaux derivatives for convex functions in linear spaces with applications. Kyungpook Math. J., 2012, vol. 52, no. 4, pp. 495-511.

DOI: https://doi.org/10.5666/kmj.2012.52.4.495

[9] Kikianty E., Dragomir S. S. Hermite-Hadamard's inequality and the p$\mathrm{HH}$-norm on the Cartesian product of two copies of a normed space. Math. Inequal. Appl., 2010, vol. 13, no. 1, pp. 1-32.

DOI: https://doi.org/10.7153/mia-13-01

[10] Kikianty E., Dragomir S. S., Cerone P. Ostrowski type inequality for absolutely continuous functions on segments in linear spaces. Bull. Korean Math. Soc., 2008, vol. 45, no. 4, pp. $763-780$.

DOI: https://doi.org/10.4134/bkms.2008.45.4.763

[11] Kikianty E., Dragomir S. S., Cerone P. Sharp inequalities of Ostrowski type for convex functions defined on linear spaces and application. Comput. Math. Appl., 2008, vol. 56, no. 9, pp. 2235-2246.

DOI: https://doi.org/10.1016/j. camwa.2008.03.059

Received July 29, 2020.

In revised form, October 09, 2020.

Accepted October 09, 2020.

Published online October 19, 2020.

Victoria University,

College of Engineering \& Science,

PO Box 14428,

Melbourne City, MC 8001, Australia.

E-mail: sever.dragomir@vu.edu.au 